\title{
The NEUT neutrino interaction simulation program library
}

\author{
Yoshinari Hayato $^{1, a}$ (D) and Luke Pickering ${ }^{2}$ (D) \\ ${ }^{1}$ ICRR, Kamioka Observatory and Kavli IPMU, The University of Tokyo, Kamioka, Gifu, Japan \\ ${ }^{2}$ Department of Physics and Astronomy, Michigan State University, East Lansing, MI, USA
}

Received 30 June 2021 / Accepted 19 September 2021 / Published online 11 October 2021

(C) The Author(s) 2021

\begin{abstract}
NEUT is a neutrino-nucleus interaction simulation program library. It can be used to simulate interactions for neutrinos with between $100 \mathrm{MeV}$ and a few $\mathrm{TeV}$ of energy. NEUT is also capable of simulating hadron interactions within a nucleus and is used to model nucleon decay and hadron-nucleus interactions for particle propagation in detector simulations. This article describes the range of interactions modelled and how each is implemented.
\end{abstract}

\section{Introduction}

NEUT is primarily a neutrino-nucleus scattering simulation program library and provides a complete model capable of predicting the observations for a wide range of neutrino scattering experiments. NEUT is capable of simulating neutrino-nucleon and coherent neutrinonucleus interactions in a number of reaction channels over a neutrino energy range from $100 \mathrm{MeV}$ to a few $\mathrm{TeV}$. Additionally, NEUT incorporates initial and final state nuclear effects for interactions with nuclei from boron to lead. One of the most important nuclear effects is the re-scattering of hadrons, which are produced in the primary neutrino-nucleon interaction, as they propagate out of the nuclear medium. This re-scattering can result in hadron absorption, extra hadron production or knock-out, or distortion of the nuclear-leaving particle kinematic spectra. The NEUT hadron re-scattering model has also been used to simulate low-energy pionnucleus scattering both to tune the model to the experimental data [1] and to simulate pion propagation in neutrino-scattering experimental simulations. Finally, NEUT can also simulate various nucleon decay channels to support experimental searches for the process.

NEUT has a long rich history, originally developed in the 1980s as a tool to study atmospheric neutrinos and nucleon decay in the Kamiokande experiment [2], and some of the original FORTRAN77 code is still in use. NEUT continues to be predominantly developed and maintained by members of the Kamiokande series of experiments (Super-Kamiokande, T2K, Hyper-Kamiokande) and many source files contain comments messages from the numerous physicists who have contributed to the simulation over the past 35 years-including those working on the Nobel prize-winning Super-Kamiokande (SK) analysis [3]. Recent development has targeted

\footnotetext{
${ }^{\mathrm{a}}$ e-mail: hayato@suketto.icrr.u-tokyo.ac.jp (corresponding author)
}

the improvements most-needed for precise and robust analyses of SK and T2K neutrino oscillation data and neutrino cross-section measurements. At T2K energies, charged-current quasi-elastic interactions dominate. It has become clear over the last decade that such interactions can only be precisely predicted by incorporating detailed models of relevant nuclear dynamics. For the multi-GeV samples used in SK analyses, shallow and deep inelastic scattering channels are critical for modelling the expected rate of multi-ring events seen in the detector. The transition region between resonance excitation and deep inelastic scattering has proven particularly difficult to model well. Because of the in-house nature of NEUT development and analysis usage, it is not yet open source. We do not yet have the resources to migrate to an open source model, but, access to the code and usage instructions are available upon request.

\section{Motivating the use of an interaction simulation}

The primary goal of atmospheric and long-baseline neutrino-scattering experiments is to study neutrino oscillation, which occurs as a function of neutrino energy and flavor. As neutrinos are neutral particles, their properties (including their energy) can only be inferred from observable secondary particles produced when they interact with matter in our particle detectors. Interaction simulations are used to predict the probability of neutrinos of a given flavor and energy to interact with a given target, the observable secondary particle spectra produced in the neutrino-nucleus interaction, and event selection efficiencies and purities. The common use of nuclear targets in neutrino scattering experiments further complicates the problem as secondary hadrons produced in the primary neutrino interaction can be absorbed or lose energy before leaving 

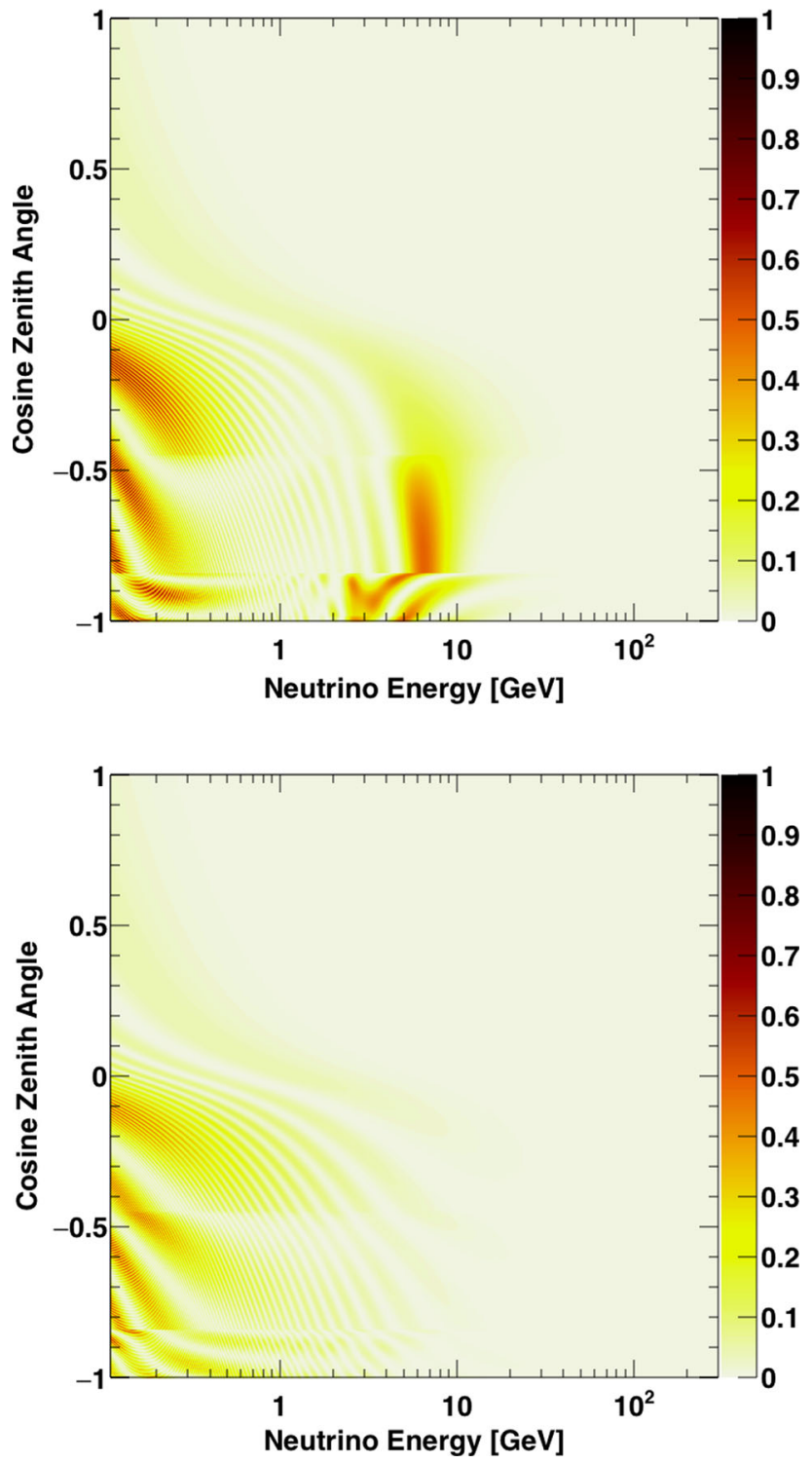

Fig. 1 Oscillation probabilities for atmospheric $\nu_{\mu}$ to $\nu_{e}$ (top) and $\bar{\nu}_{\mu}$ to $\bar{\nu}_{e}$ (bottom) as a function of neutrino energy and the cosine of the zenith angle (acting as a proxy for propagation distance through the Earth) assuming a normal neutrino mass hierarchy. Matter effects in the Earth produce distortions in oscillations of $\nu_{\mu}$ between 2 and $10 \mathrm{GeV}$, which are not seen for $\bar{\nu}_{\mu}$ oscillations. Figures reproduced from Ref. [4] The oscillation parameters used are: $\Delta m_{32}^{2}=2.5 \times$ $10^{-3} \mathrm{eV}^{2}, \sin ^{2} \theta_{23}=0.5, \sin ^{2} \theta_{13}=0.0219$, and $\delta_{C P}=0$

the target nucleus, obfuscating the details of the primary interaction. As a result, interaction simulations are a critical tool in the analysis of neutrino oscillation data. The neutrino energy range modeled most carefully by NEUT is between 0.1 and $10 \mathrm{GeV}$. This range is motivated by the typical energies for oscillation features expected in SK and T2K data, as seen in Figs. 1 and 2 .

To test oscillation hypotheses, observable distributions that correlate strongly with the neutrino energy
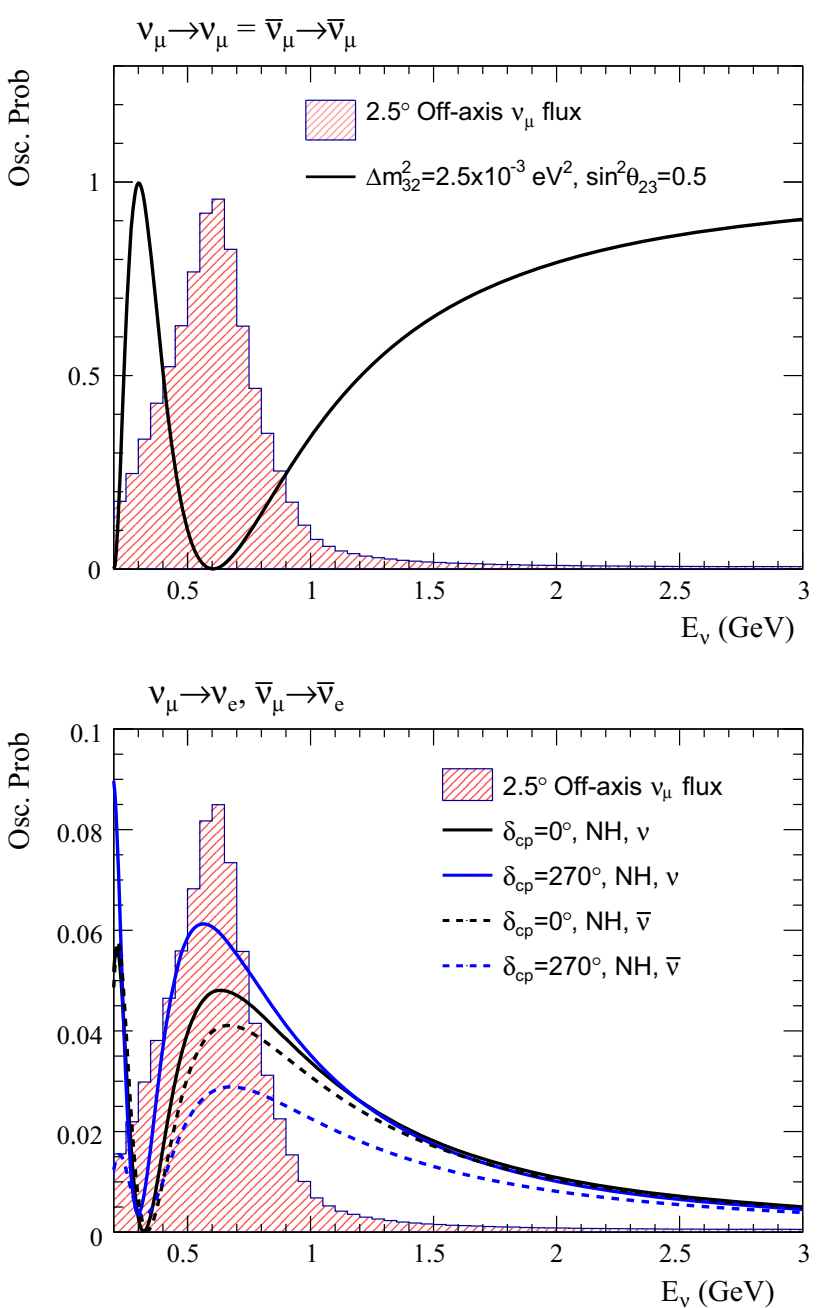

Fig. 2 The T2K $\nu_{\mu}$ flux $[5,6]$ (energy spectral shape) and overlaid oscillation probabilities for the $\nu_{\mu}$ to $\nu_{\mu}$ (top) and $\nu_{\mu}$ to $\nu_{e}$ and $\bar{\nu}_{\mu}$ to $\bar{\nu}_{e}$ (bottom) as a function of neutrino energy. The black (blue) curves correspond to minimal (maximal) values of the CP-violating phase $\delta_{\mathrm{CP}}$. The oscillation parameters used are $\Delta m_{32}^{2}=2.5 \times 10^{-3} \mathrm{eV}^{2}$, $\sin ^{2}\left(\theta_{23}\right)=0.5, \sin ^{2}\left(2 \theta_{13}\right)=0.1$

distribution are sampled and compared to simulated predictions to extract oscillation parameter constraints. An example of such an observable is

$$
E_{\mathrm{QE}}^{\mathrm{Rec}}\left(p_{\ell}, \theta_{\ell}\right)=\frac{2 M_{N, i} E_{\ell}-M_{\ell}^{2}+M_{N, f}^{2}-M_{N, i}^{2}}{2\left(M_{N, i}-E_{\ell}+p_{\ell} \cos \theta_{\ell}\right)}
$$

where $M_{N, i}, M_{N, f}$, and $M_{\ell}$ are the mass of the initial-state nucleon, final-state nucleon, and final-state charged lepton, respectively; $E_{\ell}, p_{\ell}$, and $\theta_{\ell}$ are the energy, three-momentum, and angle of the final-state charged-lepton, respectively. $E_{\mathrm{QE}}^{\mathrm{Rec}}$ is an unbiased neutrino energy estimator for the charged-current quasielastic (CCQE) reaction off a free neutron, $\nu_{\ell}+n \rightarrow$ $\ell^{-}+p$, or a free proton, $\overline{\nu_{\ell}}+p \rightarrow \ell^{+}+n$, which is only a function of the kinematics of the final-state charged lepton. All operating neutrino-scattering experiments use 

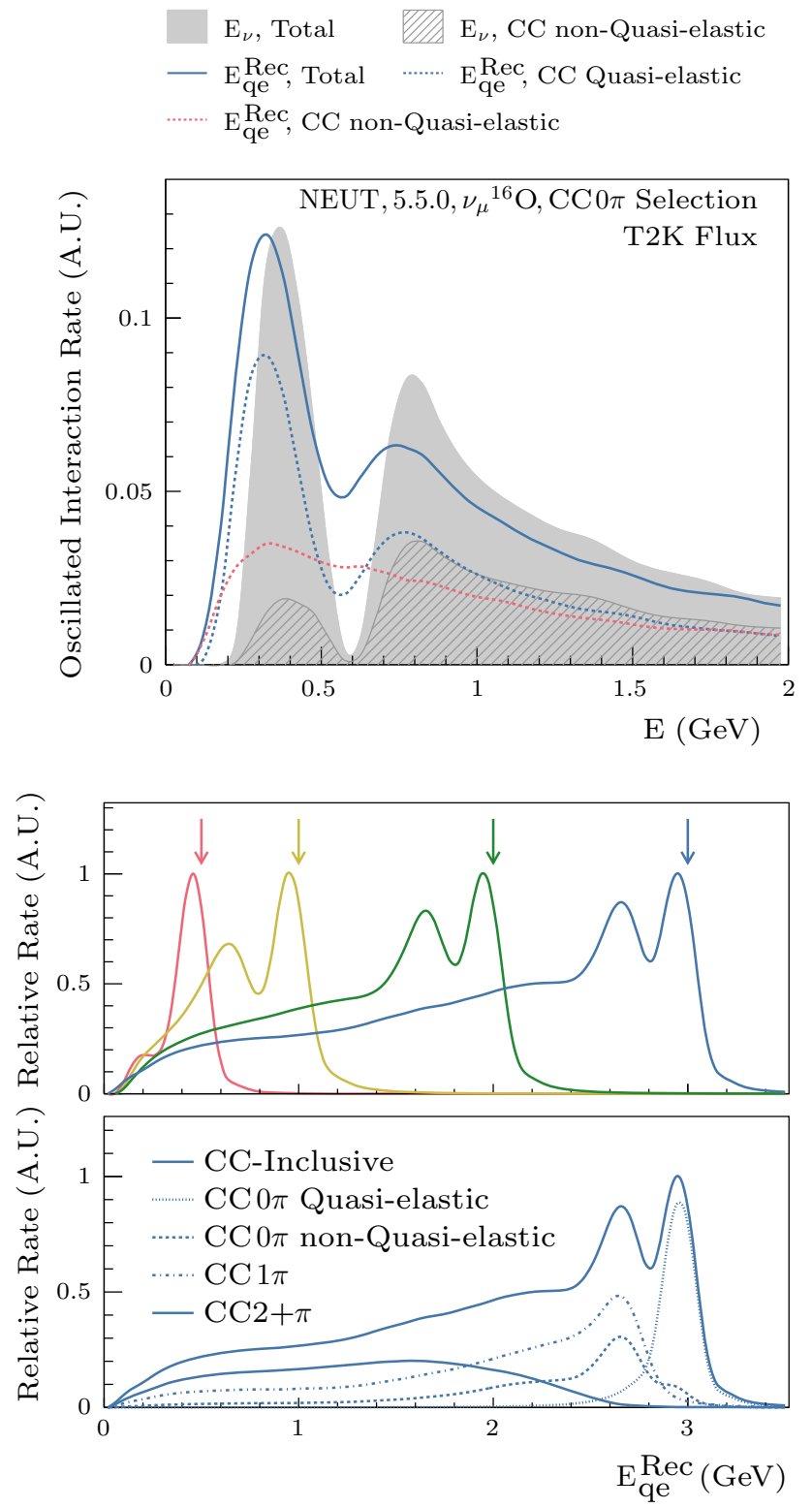

Fig. 3 (top) The true and reconstructed neutrino energy for charged-current interactions producing no observable pion in a typical oscillated $\mathrm{T} 2 \mathrm{~K} \nu_{\mu}$ muon neutrino flux at SK $[5,6]$. The reconstructed energy feed down for non-quasielastic interactions smears away the oscillation signature that can be clearly seen in the true energy spectrum. (middle) The reconstructed energy feed down for four monoenergetic muon neutrino fluxes. The colored arrows show the true energy of the corresponding reconstructed energy distribution. (bottom) The $3 \mathrm{GeV}$ distribution is separated by number of secondary mesons produced

nuclear targets, where CCQE reactions occur predominantly with bound nucleons. For such interactions, $E_{\mathrm{OE}}^{\mathrm{Rec}}$ is biased by the potential energy associated with the nucleon binding and is smeared by the Fermi motion of the nucleon confinement. ${ }^{1}$ For interactions that exhibit strong nuclear-effects or produce extra hadrons, here referred to as CC non-QE, $E_{\mathrm{QE}}^{\mathrm{Rec}}$ is in general a significant underestimate of the true neutrino energy. Figure 3 (top) shows the NEUT prediction of the oscillated rate of charged-current neutrino-oxygen interactions that produce no observable pions, a $\mathrm{CC} 0 \pi$ event topology. The CCQE and CC non-QE components are shown separately to highlight how the shape of their contributions differ between the $E_{\nu}$ and $E_{\mathrm{OE}}^{\mathrm{Rec}}$ projections. For non-QE interactions, the shape of the oscillation is largely smeared away. The CC non-QE component of the $\mathrm{CC} 0 \pi$ topology can be further broken down into interactions that either do not produce secondary pions, or do produce secondary pions that are subsequently re-absorbed before leaving the struck nucleus. Figure 3 (middle and bottom) illustrates the NEUT-predicted evolution of $E_{\mathrm{QE}}^{\mathrm{Rec}}$ for a relevant range of discrete neutrino energies. It can be seen that $E_{\mathrm{QE}}^{\mathrm{Rec}}$ exhibits significant reconstructed energy feed down above about $1 \mathrm{GeV}$, where secondary meson production is common. For higher energies, calorimetric estimators that account for hadronic energy are more accurate. However, current neutrino interaction models are often only predictive in lepton kinematics and significant uncertainties should be assigned to the predictions of hadronic particle spectra, especially for baryons and heavy mesons.

To accurately interpret observable distributions, interaction simulations are relied upon to predict the rate and observable projections for neutrino interactions over a range of energies and for a number of different target nuclei as well as the migration of these primary interactions into observable topologies. The next section details the nuclear dynamics, primary neutrino interaction, and hadronic re-scattering physics models implemented in NEUT.

The most important proton decay channels, predicted by various grand unification theories, produce a lepton and a meson, e.g. $p \rightarrow e^{+} \pi^{0}, p \rightarrow \mu^{+} \pi^{0}$, or $p \rightarrow \bar{\nu} K^{+}$. Neutrinos also often produce a lepton and one or more mesons when interacting with a nucleon. There are plenty of atmospheric neutrinos, which have sufficient energy to undergo such interactions, providing a significant background for proton decay searches. The majority of neutrino interactions also produce a baryon in addition to a lepton and a meson, but these may not be visible in detectors such as SK. The typical predicted momentum of mesons from both proton decays and atmospheric neutrino interactions are below several hundred $\mathrm{MeV} / \mathrm{c}$ and thus, the probabilities for them to scatter before leaving the nucleus is high. Accurately predicting neutrino-induced backgrounds and hadronic re-scattering within the nucleus is critical for the sensitivity of proton decay searches.

\footnotetext{
1 This effect can be seen in the offset between the initial neutrino energy arrow and the peak of the $E_{\mathrm{QE}}^{\text {Rec }}$ distribution in Fig. 3 (middle).
} 


\section{The physics in NEUT}

\subsection{Simulating an interaction}

In general, NEUT factorizes the simulation of an interaction of a neutrino with flavour, $\ell$, and energy, $E_{\nu}$, into four discrete steps. First, a specific interaction channel is chosen randomly with probability, $P=$ $\sigma_{T}^{i}\left(E_{\nu_{\ell}}\right) / \sigma_{T}^{\text {tot }}\left(E_{\nu_{\ell}}\right)$, where $\sigma_{T}^{\text {tot }}\left(E_{\nu_{\ell}}\right)$ is the total cross section and $\sigma_{T}^{i}\left(E_{\nu_{\ell}}\right)$ is the cross section for the specific target nuclei, $T$, and channel, $i$, where $i$ is an integer that identifies the interaction process and is defined in Table 1 (charged current) and Table 2 (neutral current). For neutrino-nucleon interaction channels, the nuclear-target cross section is usually constructed as $\sigma_{T}^{i}=Z \sigma_{p}^{i}+(A-Z) \sigma_{n}^{i}$, where $A$ and $Z$ are the nucleon number and the proton number of the target nuclei and $\sigma_{p}^{i}$ and $\sigma_{n}^{i}$ are the bound proton and bound neutron cross sections. For historical reasons, free protons can be added to nuclear targets to build simple molecular targets such as $\mathrm{H}_{2} \mathrm{O}$ and $\mathrm{CH}$. Figure 4 shows the NEUT water-target cross-section predictions separated into classes of interaction channel.

Second, the primary neutrino interaction, or hard scatter, is simulated. For the majority of channels, this step involves choosing a bound nucleon from an initialstate nuclear model, then choosing interaction kinematics according to the specific interaction model, and finally choosing any remaining particle kinematics not specified by the model. This step is performed under the impulse approximation [8], which treats the target bound nucleon and the remnant nucleus as evolving independently during and after the hard scatter. This further factorizes the simulation as, to first order, the sampling of the nuclear model does not depend on the interaction kinematics chosen.

For the coherent pion-production channels (Enum 16 and 36 ), the interaction occurs coherently between the neutrino and the target nucleus and as a result no bound nucleon target is chosen and this is considered the final step of the simulation. For other channels, the final state hadrons are then passed on to the third step, the nucleon and meson intra-nuclear re-scattering simulation, where hadrons can elastically scatter, exchange charge with a nucleon in the nucleus, or be produced or absorbed as they are stepped out of the nuclear medium.

Finally, for oxygen targets only, the final state nuclear remnant can be left in an excited state after the interaction and a number of nuclear de-excitations, producing low energy photons $(\mathcal{O}(1-10) \mathrm{MeV})$, are modeled following Ref. [9]. Careful treatment of the de-excitation oxygen is important for precisely simulating interactions in the sensitive SK detector.

For the majority of particles produced in the hard scatter and subsequent re-scattering, NEUT stores their properties in an event vector file that can be used as input to further experiment simulation processes. The only exceptions are tau and omega particles, which are decayed during the NEUT simulation by TAUOLA [10]

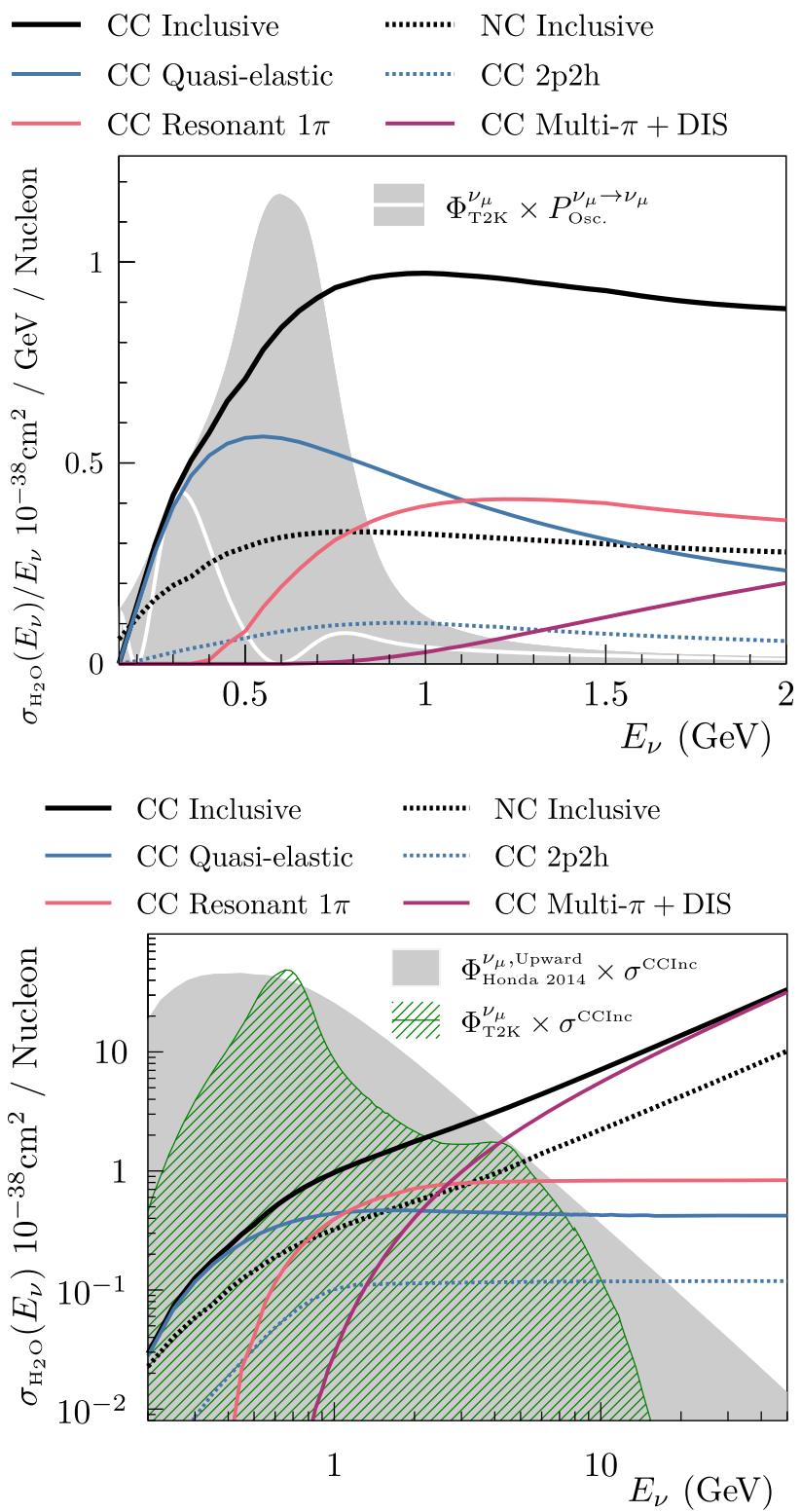

Fig. 4 The NEUT-predicted muon neutrino-water cross sections overlaid on the T2K muon neutrino flux [6], with a typical oscillation (top), and upward atmospheric muon neutrino fluxes [7] multiplied by the charged-current inclusive total cross section (bottom). The flux multiplied by the cross section is proportional to the expected interaction rate. Above $4 \mathrm{GeV}$, the expected number of interactions in SK arising from the T2K beam falls significantly faster than from atmospheric neutrinos. n.b. The cross sections presented in the top pane are divided by the neutrino energy, whereas in the bottom pane, they are not. This is to emphasise the saturation of the interaction channels associated with lower four-momentum transfer at SK energies and the sharp turn-on seen over T2K flux distribution 
Table 1 Neutrino charged-current scattering reactions simulated by NEUT, where $\mathrm{p}$ refers to a proton, $\mathrm{n}$ to a neutron, N to a neutron or proton, ${ }^{A} \mathbf{X}$ to an entire nucleus, and $W$ to the invariant mass of the final state hadronic system

\begin{tabular}{|c|c|c|c|c|}
\hline \multirow[t]{2}{*}{ Channel name } & \multirow[t]{2}{*}{ Reaction } & \multicolumn{2}{|c|}{$\sigma^{\nu_{\mu}} / 10^{-38} \mathrm{~cm}^{2} / \mathrm{N}$} & \multirow[t]{2}{*}{ Enum } \\
\hline & & $0.6 \mathrm{GeV}$ & $10 \mathrm{GeV}$ & \\
\hline \multirow[t]{2}{*}{ CCQE (1p1h) } & $\nu_{\ell}+\mathrm{n} \rightarrow \ell^{-}+\mathrm{p}$ & 0.76 & 0.95 & 1 \\
\hline & $\bar{\nu}_{\ell}+\mathrm{p} \rightarrow \ell^{+}+\mathrm{n}$ & 0.20 & 0.85 & -1 \\
\hline \multirow[t]{2}{*}{$2 \mathrm{p} 2 \mathrm{~h}$} & $\nu_{\ell}+\mathrm{Nn} \rightarrow \ell^{-}+\mathrm{Np}$ & 0.03 & 0.08 & 2 \\
\hline & $\bar{\nu}_{\ell}+\mathrm{Np} \rightarrow \ell^{+}+\mathrm{Nn}$ & 0.01 & 0.08 & -2 \\
\hline \multirow[t]{2}{*}{ CCRes $1 \pi^{+}$} & $\nu_{\ell}+\mathrm{p} \rightarrow \ell^{-}+\mathrm{p} \pi^{+}$ & 0.15 & 0.77 & 11 \\
\hline & $\nu_{\ell}+\mathrm{n} \rightarrow \ell^{-}+\mathrm{n} \pi^{+}$ & 0.03 & 0.52 & 13 \\
\hline \multirow[t]{2}{*}{ CCRes $1 \pi^{0}$} & $\nu_{\ell}+\mathrm{n} \rightarrow \ell^{-}+\mathrm{p} \pi^{0}$ & 0.04 & 0.39 & 12 \\
\hline & $\bar{\nu}_{\ell}+\mathrm{p} \rightarrow \ell^{+}+\mathrm{n} \pi^{0}$ & 0.01 & 0.31 & -12 \\
\hline \multirow[t]{2}{*}{ CCRes $1 \pi^{-}$} & $\bar{\nu}_{\ell}+\mathrm{n} \rightarrow \ell^{+}+\mathrm{n} \pi^{-}$ & 0.02 & 0.63 & -11 \\
\hline & $\bar{\nu}_{\ell}+\mathrm{p} \rightarrow \ell^{+}+\mathrm{p} \pi^{-}$ & $5 \times 10^{-3}$ & 0.41 & -13 \\
\hline CCDif1 $\pi^{+}$ & $\nu_{\ell}+\mathrm{p} \rightarrow \ell^{-}+\mathrm{p} \pi^{+}$ & B.T. & 0.03 & 15 \\
\hline $\operatorname{CCDif} 1 \pi^{-}$ & $\bar{\nu}_{\ell}+\mathrm{p} \rightarrow \ell^{+}+\mathrm{p} \pi^{-}$ & B.T. & 0.03 & -15 \\
\hline CCCoh $1 \pi^{+}$ & $\nu_{\ell}+{ }^{A} \mathbf{X} \rightarrow \ell^{-}+{ }^{A} \mathbf{X}+\pi^{+}$ & $1 \times 10^{-3}$ & 0.04 & 16 \\
\hline $\operatorname{CCCoh} 1 \pi^{-}$ & $\bar{\nu}_{\ell}+{ }^{A} \mathbf{X} \rightarrow \ell^{+}+{ }^{A} \mathbf{X}+\pi^{-}$ & $1 \times 10^{-3}$ & 0.04 & -16 \\
\hline \multirow[t]{2}{*}{ CCRes $1 \gamma$} & $\nu_{\ell}+\mathrm{n} \rightarrow \ell^{-}+\mathrm{p} \gamma$ & $2 \times 10^{-4}$ & $2 \times 10^{-3}$ & 17 \\
\hline & $\bar{\nu}_{\ell}+\mathrm{p} \rightarrow \ell^{+}+\mathrm{n} \gamma$ & $3 \times 10^{-5}$ & $1 \times 10^{-3}$ & -17 \\
\hline \multirow[t]{2}{*}{$\mathrm{CCN} \pi$} & $\nu_{\ell}+\mathrm{N} \rightarrow \ell^{-}+\mathrm{N}^{\prime}+x \pi$ & B.T. & 0.85 & 21 \\
\hline & $\begin{array}{l}\bar{\nu}_{\ell}+\mathrm{N} \rightarrow \ell^{+}+\mathrm{N}^{\prime}+x \pi \\
\text { where } x>1 \text { and } 1.3<W<2.0 \mathrm{GeV}\end{array}$ & B.T. & 0.62 & -21 \\
\hline \multirow[t]{2}{*}{ CCRes $1 \eta^{0}$} & $\nu_{\ell}+\mathrm{n} \rightarrow \ell^{-}+\mathrm{p} \eta^{0}$ & B.T. & 0.19 & 22 \\
\hline & $\bar{\nu}_{\ell}+\mathrm{p} \rightarrow \ell^{+}+\mathrm{n} \eta^{0}$ & B.T. & 0.14 & -22 \\
\hline CCRes $1 K^{0}$ & $\nu_{\ell}+\mathrm{n} \rightarrow \ell^{-}+\Lambda+K^{+}$ & B.T. & 0.06 & 23 \\
\hline CCRes $1 K^{+}$ & $\bar{\nu}_{\ell}+\mathrm{p} \rightarrow \ell^{+}+\Lambda+K^{0}$ & B.T. & 0.03 & -23 \\
\hline \multirow[t]{2}{*}{ CCDIS } & $\nu_{\ell}+\mathrm{N} \rightarrow \ell^{-}+\mathrm{N}^{\prime}+x \pi$ & B.T. & 4.53 & 26 \\
\hline & $\begin{array}{l}\bar{\nu}_{\ell}+\mathrm{N} \rightarrow \ell^{+}+\mathrm{N}^{\prime}+x \pi \\
\text { where } W>2.0 \mathrm{GeV}\end{array}$ & B.T. & 1.30 & -26 \\
\hline
\end{tabular}

The $\nu_{\mu}$ and $\overline{\nu_{\mu}}$ cross sections per nucleon for each process at $600 \mathrm{MeV}$ and $10 \mathrm{GeV}$ are included, where B.T. stands for below threshold and highlights kinematically disallowed channels for $600 \mathrm{MeV}$ neutrinos. For reference, the NEUT reaction identifier enumeration is included. CCQE (1p1h) cross sections are for bound nucleon in oxygen and calculated using the spectral function model. Coherent pion production cross-sections are also for oxygen

and a custom omega decay simulation code, respectively. The final states of such decays are written to the event vector.

\subsection{The hard scatter}

This section details the neutrino scattering physics models implemented in NEUT, roughly ordered by the degree of inelasticity of the interaction.

\subsubsection{Quasi-elastic scattering}

The cross-section of neutrino-nucleon charged-current quasi-elastic (CCQE) interaction was formalized by Llewellyn Smith [11]. In some of the literature, an equivalent channel is referred to as $1 \mathrm{p} 1 \mathrm{~h}$, for oneparticle one-hole. Three different nuclear models are implemented in NEUT for simulating CCQE interactions, the relativistic global Fermi-gas (GRFG) model, the local Fermi-gas (LFG) model and the spectral function (SF) model.
The NEUT implementation of the GRFG cross section follows the prescription by Smith and Moniz [12]. The LFG implementation uses the model by Nieves et al. [13] and includes an updated removal energy treatment implemented by Bourguille et al. [14]. Simple Fermi-gas models tend to over-predict the cross section for forward going leptons, as a result this model takes into account long- and short-range correlations of nucleons using the random phase approximation, suppressing the cross section for low 4-momentum transfer [15]. The NEUT SF uses the spectral function by Benhar et al. [16] and the implementation is based on the one in NuWro [17] with additional improvements by Furmanski [18].

These three nuclear models differ in their treatment of the bound nucleon momentum and removal energy distributions and whether there are correlations between them. Figure 5 shows the projections of interactions simulated with each of the three models into missing momentum $\left(p_{\text {miss }}\right)$ and missing energy $\left(E_{\text {miss }}\right)$, which are observable quantities for analogous measurements of electron-nucleus scattering. For the $\nu_{\ell}+{ }^{16} \mathrm{O} \rightarrow \ell^{-}+p+{ }^{15} \mathrm{O}$ interaction, $p_{\text {miss }}$ and $E_{\text {miss }}$ 
Table 2 Neutrino neutral-current scattering reactions simulated by NEUT, where p refers to a proton, $\mathrm{n}$ to a neutron, $\mathrm{N}$ to a neutron or proton, ${ }^{A} \mathbf{X}$ to an entire nucleus, and $W$ to the invariant mass of the final state hadronic system

\begin{tabular}{|c|c|c|c|c|}
\hline Channel name & Reaction & $\sigma^{\nu \mu} / 10^{-3}$ & & Enum \\
\hline & & $0.6 \mathrm{GeV}$ & $10 \mathrm{GeV}$ & \\
\hline $\operatorname{NCRes} 1 \pi^{0}$ & $\nu_{\ell}+\mathrm{n} \rightarrow \nu_{\ell}+\mathrm{n} \pi^{0}$ & 0.03 & 0.15 & 31 \\
\hline & & $9 \times 10^{-3}$ & 0.13 & -31 \\
\hline & $\nu_{\ell}+\mathrm{p} \rightarrow \nu_{\ell}+\mathrm{p} \pi^{0}$ & 0.03 & 0.15 & 32 \\
\hline & & $9 \times 10^{-3}$ & 0.12 & -32 \\
\hline NCRes $1 \pi^{0}$ & $\nu_{\ell}+\mathrm{n} \rightarrow \nu_{\ell}+\mathrm{p} \pi^{-}$ & 0.02 & 0.13 & 33 \\
\hline & & $5 \times 10^{-3}$ & 0.11 & -33 \\
\hline NCRes $1 \pi^{+}$ & $\nu_{\ell}+\mathrm{p} \rightarrow \nu_{\ell}+\mathrm{n} \pi^{+}$ & 0.02 & 0.12 & 34 \\
\hline & & $5 \times 10^{-3}$ & 0.10 & -34 \\
\hline $\operatorname{NCDif} 1 \pi^{0}$ & $\nu_{\ell}+\mathrm{p} \rightarrow \nu_{\ell}+\mathrm{p} \pi^{0}$ & B.T. & 0.01 & 35 \\
\hline & & B.T. & 0.01 & -35 \\
\hline $\operatorname{NCCoh} 1 \pi^{0}$ & $\nu_{\ell}+{ }^{A} \mathbf{X} \rightarrow \nu_{\ell}+{ }^{A} \mathbf{X}+\pi^{0}$ & $1 \times 10^{-3}$ & 0.02 & 36 \\
\hline & & $1 \times 10^{-3}$ & 0.02 & -36 \\
\hline NCRes1 $\gamma$ & $\nu_{\ell}+\mathrm{n} \rightarrow \nu_{\ell}+\mathrm{n} \gamma$ & $2 \times 10^{-4}$ & $1 \times 10^{-3}$ & 38 \\
\hline & & $6 \times 10^{-5}$ & $8 \times 10^{-4}$ & -38 \\
\hline & $\nu_{\ell}+\mathrm{p} \rightarrow \nu_{\ell}+\mathrm{p} \gamma$ & $2 \times 10^{-4}$ & $9 \times 10^{-4}$ & 39 \\
\hline & & $6 \times 10^{-5}$ & $8 \times 10^{-4}$ & -39 \\
\hline $\mathrm{NCN} \pi$ & $\nu_{\ell}+\mathrm{N} \rightarrow \nu_{\ell}+\mathrm{N}^{\prime}+x \pi$ & $2 \times 10^{-4}$ & 0.25 & 41 \\
\hline & & $3 \times 10^{-5}$ & 0.23 & -41 \\
\hline & where $x>1$ and $1.3<W<2.0 \mathrm{GeV}$ & & & \\
\hline NCRes $1 \eta^{0}$ & $\nu_{\ell}+\mathrm{n} \rightarrow \nu_{\ell}+\mathrm{n} \eta^{0}$ & B.T. & 0.04 & 42 \\
\hline & & B.T. & 0.03 & -42 \\
\hline & $\nu_{\ell}+\mathrm{p} \rightarrow \nu_{\ell}+\mathrm{p} \eta^{0}$ & B.T. & 0.03 & 43 \\
\hline & & B.T. & 0.02 & -43 \\
\hline NCRes $1 K^{0}$ & $\nu_{\ell}+\mathrm{n} \rightarrow \nu_{\ell}+\Lambda+K^{0}$ & B.T. & 0.01 & 44 \\
\hline & & B.T. & $7 \times 10^{-3}$ & -44 \\
\hline NCRes $1 K^{+}$ & $\nu_{\ell}+\mathrm{p} \rightarrow \nu_{\ell}+\Lambda+K^{+}$ & B.T. & $9 \times 10^{-3}$ & 45 \\
\hline & & B.T. & $7 \times 10^{-3}$ & -45 \\
\hline NCDIS & $\nu_{\ell}+\mathrm{N} \rightarrow \nu_{\ell}+\mathrm{N}^{\prime}+x \pi$ & B.T. & 1.37 & 46 \\
\hline & & B.T. & 0.49 & -45 \\
\hline & where $W>2.0 \mathrm{GeV}$ & & & \\
\hline NCEL (1p1h) & $\nu_{\ell}+\mathrm{n} \rightarrow \nu_{\ell}+\mathrm{n}$ & 0.13 & 0.16 & 51 \\
\hline & & 0.05 & 0.16 & -51 \\
\hline & $\nu_{\ell}+\mathrm{p} \rightarrow \nu_{\ell}+\mathrm{p}$ & 0.16 & 0.21 & 52 \\
\hline & & 0.06 & 0.19 & -52 \\
\hline
\end{tabular}

The $\nu_{\mu}$ and $\overline{\nu_{\mu}}$ cross sections per nucleon for each process at $600 \mathrm{MeV}$ and $10 \mathrm{GeV}$ are included, where B.T. stands for Below Threshold and highlights kinematically disallowed channels for $600 \mathrm{MeV}$ neutrinos. For each reaction, the first line shows the cross-section for neutrinos and the second line shows the cross-section for anti-neutrinos. For reference, the NEUT reaction identifier enumeration is included. NCEL $(1 \mathrm{p} 1 \mathrm{~h})$ cross sections are for bound nucleon in oxygen and calculated using the spectral function model. Coherent pion production cross sections are also for oxygen

are defined as

$$
\begin{aligned}
p_{\mathrm{miss}} & =\left|\mathbf{p}_{\nu}-\mathbf{p}_{\ell}-\mathbf{p}_{p}\right| \\
T_{15 \mathrm{O}} & =\sqrt{p_{\mathrm{miss}}^{2}+M_{15}^{2} \mathrm{O}}-M_{15 \mathrm{O}}, \\
E_{\mathrm{miss}} & =E_{\nu}+M_{n}-E_{\ell}-E_{p}-T_{15 \mathrm{O}},
\end{aligned}
$$

where $T_{15} \mathrm{O}$ is the reconstructed kinetic energy and $M_{15} \mathrm{O}$ is the ground-state mass of the nuclear remnant, which in this case is an unstable isotope but is long-lived on the timescale of the impulse approximation. These quantities are of interest because $E_{\text {miss }}$ is approximately the energy lost to the nuclear response during the interaction and $p_{\text {miss }}$ is the momentum of the struck nucleon in the lab frame. It is clear from Fig. 5 that the different nuclear models make different predictions about their distribution and correlation, notably the ${ }^{16} \mathrm{O}$ shell structure is visible in the SF predictions. The SF model is tuned to exclusive electron-nucleus scattering data and is only available for a subset of target nuclei, and in NEUT is only implemented for ${ }^{12} \mathrm{C},{ }^{16} \mathrm{O}$, and ${ }^{56} \mathrm{Fe}$, the most important nuclei for SK and T2K analyses. For interactions with other nuclei, such as ${ }^{40} \mathrm{Ar}$, only the GRFG or the LFG nuclear models are implemented.

Beyond the choice of nuclear models, the vector and axial-vector nucleon form factors control the strength and shape of quasi-elastic interactions. There are two vector form factors of nucleon implemented in NEUT, 

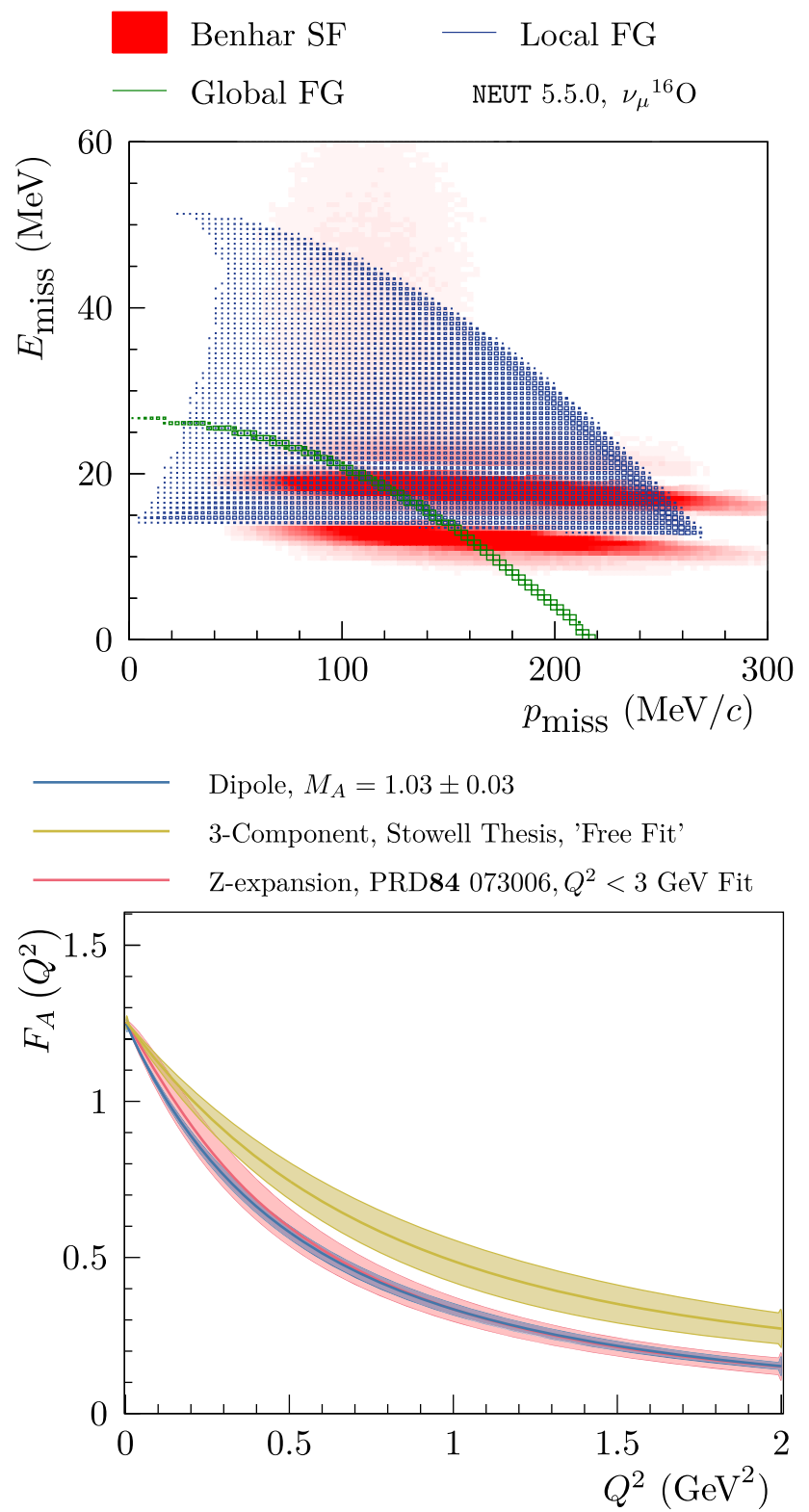

Fig. 5 (Top) The reconstructed missing energy and missing momentum distributions for the three initial-state nuclear models implemented for the CCQE channel. (Bottom) Three nucleon axial form factor models and their associated uncertainties derived in Ref. [24] (dipole and 3component) and Ref. [22] (Z-expansion)

the simple dipole form factor and BBBA05 [19]. By default, BBBA05 is used as this form factor was developed to reproduce experimental electron-scattering data.

There are four axial nucleon form factor models implemented, the dipole form factor, BBBA07 [20], the Z-expansion model [21] and the 3-component model [24]. The 3-component model is inspired by the 2 -component model [23] and was created to provide additional shape freedom by expanding the 2-component model, which quickly decays with four-momentum transfer squared,
$Q^{2}$. This model has the freedom to vary the gradient of the form factor at low- $Q^{2}$ leaving the free parameters in the 2-component model to set the shape at higher momentum transfer. The 3-component model is able to be continuously varied between the shape of both the 2-component and the simple dipole model. Figure 5 presents the shape of three of the axial form factor models with associated uncertainties derived fits to hydrogen and deuterium bubble chamber data. The dipole and 3-component model uncertainties are reproduced from Stowell [24], and the Z-expansion model was fit by Meyer et al. [22].

For neutral current elastic (NCEL) scattering, the treatment and available model components are equivalent to CCQE, except that an LFG initial-state model is not implemented for NCEL.

\subsubsection{Charged current multi-nucleon scattering}

A number of accelerator-based neutrino oscillation experiments, K2K, MINOS, and MiniBooNE, started taking high-statistics neutrino-nucleus scattering data in the 2000s. These experiments found that the number of the observed CCQE-like (equivalent to the $\mathrm{CC} 0 \pi$ topology introduced earlier) events were a few tens of percent larger than predicted by the models, but with a relative deficit of very forward-going muons [25-27]. One of the sources of these discrepancies was thought to be coming from neutrino-nucleus interaction channels, which were not implemented in the simulations used. The most-probable candidate is now believe to be the so-called multi-nucleon interaction, of which a similar process is known to exist in electron-nucleus scattering. Inclusion of this interaction into neutrino-nucleus simulations was discussed by Marteau in 1999 [28].

In NEUT, the Valencia model by Nieves et al. [13] is implemented. This model considers an interaction involving the production of two nucleons and two holes in a ground-state nuclear target (often called a $2 \mathrm{p} 2 \mathrm{~h}$ interaction in the literature). Their model includes processes involving the exchange of mesons between two nucleons and thus, sometimes, it is often referred to as a Meson Exchange Current, or MEC, model. The model is not applicable for large momentum transfer and thus the three-momentum transfer $\left(q_{3}\right)$ to the nucleus is limited to $q_{3}<1.2 \mathrm{GeV} / \mathrm{c}$. This model does not predict how the four-momentum is distributed between the two final-state nucleons, NEUT follows the implementation in NuWro [17]. The directions of the outgoing nucleons are selected to be uniformly distributed in the center of mass frame of the nucleons. A separation energy (sometimes ambiguously referred to as the binding energy) is subtracted from the energy transfer from the lepton system. The outgoing nucleon momenta are required to be larger than the local Fermi surface momentum at the interaction position within the nucleus. The model for the binding energy is described in Ref. [14] and depends on the interaction position in the nucleus. For oxygen, typically between $\sim 50$ and $\sim 75 \mathrm{MeV}$ of energy is lost to the nuclear response. 
Neutral current multi-nucleon scattering is not yet implemented in NEUT.

\subsubsection{Single meson and gamma productions}

Single pion production is one of the dominant neutrino interaction channels in the few-GeV energy region. Therefore, it is important to understand these interactions to study neutrino oscillations using the atmospheric or the accelerator neutrinos. The particles from the single meson productions are similar to the ones from nucleon decay, and thus, these interactions are also important in the nucleon decay searches.

Single pion production in NEUT is implemented following the model by Rein and Sehgal [29]. An improved model, which takes into account the lepton mass correction, by Berger and Sehgal [30] is also implemented. Both of the models simulate these interactions in two steps. First, a neutrino excites the nucleon and produce intermediate baryon resonance state, which then decays into a single meson or gamma and baryon. The range of the invariant mass of the intermediate state, $W$, is between the pion-production threshold and smaller than $2 \mathrm{GeV} / \mathrm{c}^{2}$. In these two models, two kinds of form factors are implemented. The first is a simple dipole form, as used in the original publications by Rein and Sehgal [29]. The axial vector mass of the dipole type form factor was selected to be $1.21 \mathrm{GeV} / \mathrm{c}^{2}$ based on fits to K2K data. The second was formalized by Graczyk and Sobczyk [31] based on the Rarita-Schwinger formalization. The free parameters of Graczyk-Sobczyk form were extracted by fits to hydrogen and deuterium bubble chamber data. The direction of the pions in the resonance rest frame, the so-called Adler frame, can be determined using the full prescription by Rein [32] for all implemented resonances. Alternatively, the Rein calculation can be only used for interactions producing an intermediate $\Delta(1232)$ while, for higher order resonances, final-state pions are distributed uniformly in the Adler frame. For nuclear-target interactions, Pauliblocking is considered and final-state nucleon produced in the resonance decay is required to have the momentum larger than the local Fermi surface momentum modelled by an LFG. The overall effect is small, typically less than a few percent of the events are rejected.

Single Kaon, Eta and $\gamma$ productions are simulated using the same framework for the single pion production. The main differences are the decay probabilities (branching ratios) of each simulated resonance to the relevant final state. The production of other mesons, such as the omega, is not simulated with the model described in this section. However, such particles can be produced during the hadronization simulation in the Deep Inelastic Scattering reactions, described in the next section.

For neutrino interactions with nucleon bound within a nucleus, produced hadrons undergo the final state interactions as described in Sect. 3.3. Additionally, formation zone effects are taken into account as described in Sect. 3.2.6.

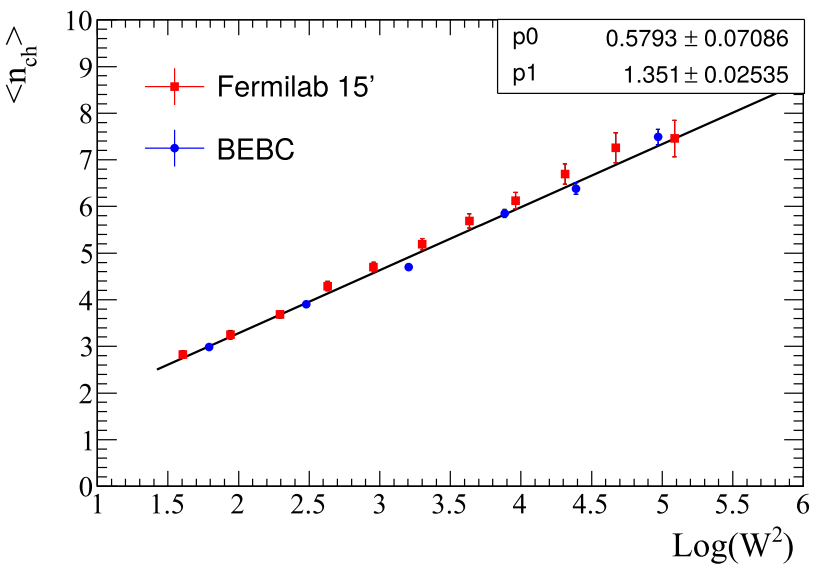

Fig. 6 Results of the fit of the averaged charged hadron multiplicities as a function of $W$ using the bubble chamber data sets [36,37]. Figure reproduced from Ref. [38]

\subsubsection{Shallow and deep inelastic scattering}

Shallow and deep inelastic scattering processes are separated as shown in Table 3 to avoid double counting single- and multi-meson production channels.

Interactions producing more than one meson is simulated with a custom multi-pion-production model for $W<2 \mathrm{GeV} / \mathrm{c}^{2}$. When $W$ is larger than $2 \mathrm{GeV} / \mathrm{c}^{2}$, all the interactions, which produced at least one meson are simulated by PYTHIA v5.72 [33], included in CERNLIB 2005. For both cases, same parton distribution functions (PDFs) are used. The default PDF is a modified version of GRV98 [34] that is based on the Bodek and Yang model [35]. The multi-pion production cross section is obtained using the function of the pion multiplicity, which itself is a function of $W$, that models the probability to produce more than one pion, with $W<2 \mathrm{GeV} / c^{2}$ region. The enforcement of multiple final-state pions avoids overlap with the resonance single meson production channels. The mean multiplicity of charged hadrons is estimated from the results of Fermilab 15-foot hydrogen bubble chamber experiment [36] and the Big European Bubble Chamber (BEBC) experiment [37]. Two parameter sets are available. The first uses the data from [36] and predicts

$$
\left\langle\mathrm{n}_{\mathrm{ch}}\right\rangle=0.09+1.83 \times \ln \left(W^{2}\right),
$$

for all channels. The second uses the fit results from both [36] and [37]. This time, the parameter sets were obtained by Bronner [38] and each combination of $\nu+$ $p, \nu+n, \bar{\nu}+p$ and $\bar{\nu}+n$ was separately fitted using the measured average charged hadron multiplicity data independently. The Bronner model for the $\nu+p$ channel is reproduced in Fig. 6 . The obtained parameter sets are 
Table 3 Shallow and deep inelastic scattering implementation in NEUT

\begin{tabular}{lll}
\hline & 1 meson & More than 1 meson \\
\hline$W<2 \mathrm{GeV} / \mathrm{c}^{2}$ & (covered by single meson production) & Custom multi-pion production model \\
$W>2 \mathrm{GeV} / \mathrm{c}^{2}$ & Pythia v5.72, included in CERNLIB 2005 & Pythia v5.72, included in CERNLIB 2005 \\
\hline
\end{tabular}

$W$ is the invariant mass of the intermediate hadron system

$$
\begin{aligned}
& \left\langle\mathrm{n}_{\mathrm{ch}}\right\rangle=0.58+1.35 \times \ln \left(W^{2}\right) \text { for } \nu+p, \\
& \left\langle\mathrm{n}_{\mathrm{ch}}\right\rangle=0.35+1.24 \times \ln \left(W^{2}\right) \text { for } \nu+n, \\
& \left\langle\mathrm{n}_{\mathrm{ch}}\right\rangle=0.41+1.18 \times \ln \left(W^{2}\right) \text { for } \bar{\nu}+p, \\
& \left\langle\mathrm{n}_{\mathrm{ch}}\right\rangle=0.80+0.94 \times \ln \left(W^{2}\right) \text { for } \bar{\nu}+n .
\end{aligned}
$$

For such interaction, we assume KNO scaling to determine the value of $W$ [39]. The forward-backward asymmetry of the charged hadron multiplicity in the hadronic center of mass system is modelled as

$$
\frac{\mathrm{n}_{\mathrm{ch}}^{F}}{\mathrm{n}_{\mathrm{ch}}^{B}}=\frac{0.35+0.95 \times \ln \left(W^{2}\right)}{0.5+0.225 \times \ln \left(W^{2}\right)}
$$

which was derived from BEBC data [40].

The cross section for the events with $W$ larger than $2 \mathrm{GeV} / \mathrm{c}^{2}$ is calculated without the multiplicity factor because there is no overlap with other implemented models. The kinematics of the produced particles are determined by PYTHIA.

For neutrino interactions with a nucleon bound within a nucleus, final state hadron interactions and formation zone effects are taken into account as for single meson production.

\subsubsection{Coherent and diffractive pion productions}

There are two coherent neutrino-nucleus pion production models implemented in NEUT. The default model is based on the prescription by Berger and Sehgal [41]. This is an update to the previously published model by Rein and Sehgal [42], which is also implemented in NEUT. The new model uses improved elastic pioncarbon cross-section data and lepton-mass effects are properly taken into account. With these improvements, the model is applicable to neutrinos with energies below several GeV.

There is a similar process called diffractive pion production, which involves coherent-like pion production, but with a single proton. NEUT implements the prescription by Rein [43].

Pions produced through these channels are not affected by the final state re-scattering, which is described in the following Sect. 3.3.1.

\subsubsection{Formation zone}

The idea of a formation zone is implemented and the production positions of hadrons in nucleus are shifted from the initial neutrino interaction position.
The implemented model in NEUT is based on SKAT data $[44,45]$. The production points of the hadrons for those interactions are shifted using the formation length $\left(L_{\mathrm{FZ}}\right)$, where

$$
L_{\mathrm{FZ}}=p / \mu^{2},
$$

$p$ is the momentum of the hadron and $\mu=0.08\left(\mathrm{GeV} / \mathrm{c}^{2}\right)$. The actual size of the shift is determined as $L_{\mathrm{FZ}} \times$ $(-\log (\operatorname{rand}[0,1])$, where rand $[0,1]$ is a random number from 0 to 1 . The distribution of secondary hadron production position is shifted further from the center of the nucleus, the produced position of hadrons shifts to the outer region of the nucleus and thus reduce the probability of final state interaction.

\subsection{Final-state hadronic re-scattering}

NEUT simulates the interactions of pions, kaons, etas, omegas, protons and neutrons, produced via neutrino interactions or nucleon decay, within the nucleus. To simulate these hadron interactions, a custom semiclassical intranuclear cascade (INC) model is used, as in most other neutrino-nucleus simulations. In NEUT, a hadron produced in the nucleus is tracked step by step from the production point until the particle escapes from the nucleus. The size of each step is fixed at $0.2 \mathrm{fm}$. At each step, it is decided whether the particle has interacted or not using the mean free paths for the modelled interaction channels. A Woods-Saxon nucleon density function and the local Fermi-gas model are used to determine the interaction positions and kinematics of the initial and final states.

\subsubsection{Meson interactions in nucleus}

Among the modelled mesons, pion is the most important in the analyses of SK and T2K. The mean free paths for the pion interactions in the Delta region are calculated following the prescriptions by Salcedo et al. [46]. Their model takes into account the in-medium correction of $\Delta$ self energy and uses the local Fermigas model. Therefore, the obtained mean free paths are expressed as functions of nuclear density and pion momentum. This model is applicable for the pions with momenta smaller than $500 \mathrm{MeV} / \mathrm{c}$. The mean free paths for pions with momenta larger $500 \mathrm{MeV} / \mathrm{c}$ was motivated by fits to pion-nucleon scattering data and are expressed as functions of pion momentum only.

In total six types of pion scattering channels are defined: low momentum pion quasi-elastic scattering, low momentum pion charge exchange interaction, pion 


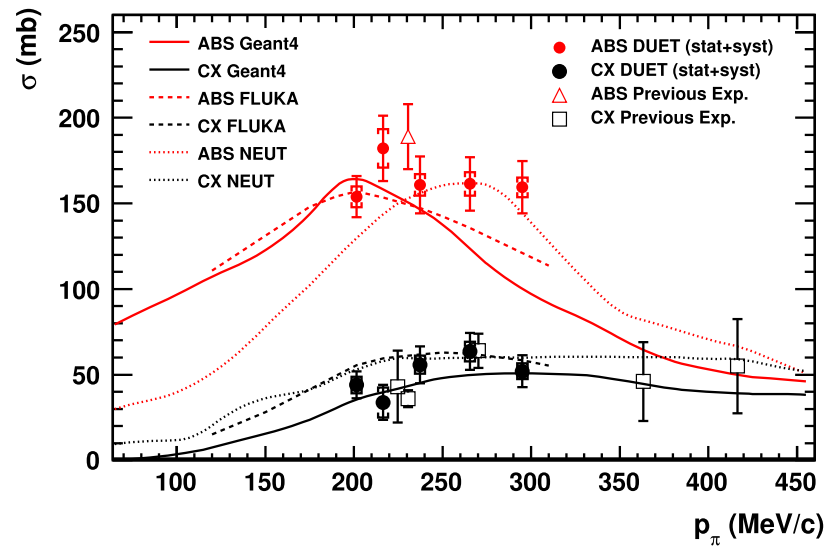

Fig. 7 Comparisons of the $\pi^{+}$absorption and charge exchange in carbon cross-section data and the simulated results of three simulation programs, GEANT4 (solid line), FLUKA (dashed line), and NEUT (dotted line). The data are taken from [47-51] and ABS (red) and CX (black). This figure is taken from Ref. [1]

absorption, high momentum pion quasi-elastic scattering, high momentum pion charge exchange interaction, high momentum pion inelastic scattering (pion production). Energy-independent normalization factors were defined for each of these six channels and fit to pionnucleus scattering data from various experiments by Pinzon Guerra et al. [1], covering pion energies up to 2 $\mathrm{GeV}$. Figure 7 shows the comparison between the pion scattering data and the simulated results from NEUT and the other simulation. The kinematics of particles after the interaction are determined using the results of the phase shift analysis [52] with medium corrections as suggested by Seki et al. [53].

The interactions of kaons, etas and omegas are treated similarly as high momentum pions. The mean free paths are provided as functions of momentum for each meson and the local Fermi-gas model is used to determine the kinematics when an interaction occurs.

For kaons, (quasi-)elastic scattering and charge exchange interactions are simulated. The mean free paths of these kaon interactions are extracted from the results of the phase shift analysis of kaon scattering data $[54,55]$. These results are also used to determine the kinematics of outgoing particles.

For etas, the $\eta N \rightarrow \eta N, \eta N \rightarrow \pi N^{\prime}$, and $\eta N \rightarrow$ $\pi \pi N^{\prime}$ processes are implemented. These interactions are simulated assuming that an eta produces an excited nucleon state, which then decays to give final state. The $N(1540), N(1650)$ intermediate baryon resonances are considered. The production cross section of baryon resonances are given by the Breit-Wigner formula:

$$
\sigma(k)=\frac{\pi}{k^{2}} \cdot \frac{\Gamma_{\eta N} \Gamma_{X}}{\left(W-M_{N}^{*}\right)^{2}+\Gamma_{\mathrm{tot}}^{2} / 4},
$$

where $W$ and $M_{N}^{*}$ are the invariant mass of the intermediate baryon resonance and the mean mass of the resonance respectively, $\Gamma_{\text {tot }}$ is the total width of $N^{*}$,
$\Gamma_{\eta N}$ is the partial width of $N^{*} \rightarrow \eta N, \Gamma_{X}$ is the partial width to the final state $X$, where $X$ is the final state meson, $\eta, \pi$ or $\pi \pi$. The produced particles are ejected isotropically in the intermediate resonance rest frame.

For omegas, the $\omega N \rightarrow \pi N, \omega N \rightarrow \rho N, \omega N \rightarrow$ $\rho \pi N^{\prime}, \omega N \rightarrow \rho \pi \pi N^{\prime}, \omega N \rightarrow \omega N$, and $\omega N \rightarrow \sigma\left(f_{0}\right) N^{\prime}$ processes are considered. The total and differential cross-sections of each channel were calculated following the prescription by Lykasov et al. [56].

\subsubsection{Nucleon interactions in nucleus}

The implementation of nucleon scattering is also based on the INC model. Three types of interaction are considered, elastic scattering and one or two pion productions. These are implemented following the work by Bertini et al. [57], for MECC-7. The same nuclear density function and local Fermi-gas model are used as the meson interactions in nucleus. When calculating the interaction kinematics, an effective nucleon mass $\left(M_{N}^{\text {eff }}\right)$ is used instead of the free mass $\left(M_{N}^{\text {free }}\right)$. Here, $M_{N}^{\text {eff }}$ is defined as

$$
M_{N}^{\text {eff }}=\sqrt{\left(M_{N}^{\text {free }}-8 \mathrm{MeV} / c^{2}\right)^{2}-\left(p_{F}^{\text {surf }}\right)^{2}},
$$

where $P_{F}^{\text {surf }}$ is the density-dependent local Fermi surface momentum. The momentum of the nucleon after an interaction is required to be larger than $P_{F}^{\text {surf }}$, which is the standard Pauli-blocking procedure in the local Fermi-gas model framework. Produced pions are assumed to arise from the decay of $\Delta(1232)$ resonances produced by nucleon-nucleon scattering. To simulate the $\Delta(1232)$ production a simple isobar model [58] is used. When a pion is produced in the nucleus after the nucleon re-scattering, that pion is independently tracked from the point of generation using the pion transport simulation described in Sect. 3.3.1. The predicted cross-sections for proton-carbon scattering is shown in Fig. 8.

\subsection{Known implementation limitations}

NEUT is primarily developed to enable world-leading SK and $\mathrm{T} 2 \mathrm{~K}$ neutrino oscillation, proton decay, and neutrino cross-section measurements, for which a fully consistent description of Nature is desirable but not necessary, and as such has some known limitations beyond the imperfect predictions of the implemented physics models.

The most prominent limitation is that the modelling of the initial nuclear state is tied to the individual interaction channel being simulated. For most channels, the initial state is always modelled as a Fermigas, for multi-nucleon scattering an LFG is used, and only for quasi-elastic scattering has the more sophisticated spectral function as an option. The SF is only designed to model QE interactions, and only for specific nuclei where relevant electron-scattering data exist, for other nuclear targets a Fermi-gas is used. While, as 


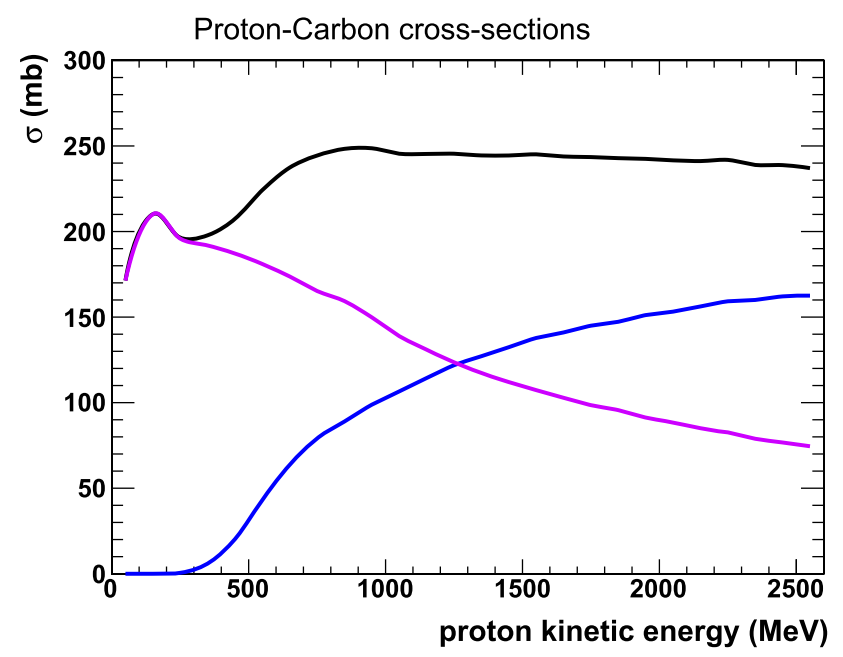

Fig. 8 Proton-carbon scattering cross-sections as a function of the proton kinetic energy, as predicted by NEUT. To obtain this plot, protons are injected from outside of the nucleus and NEUT simulates the interactions in the carbon nucleus. The black line shows the total cross section, the purple line shows the elastic scattering cross section and the blue line shows the inelastic scattering cross sections

noted above, the modelling of hadron re-scattering is based on the density and momentum predictions from an LFG model. Such an inconsistent model is sometimes affectionately referred to as a Franken-model, after the fictional scientist and his Gothic horror implementation. For single meson production, nuclear effects beyond the initial-state nuclear momentum and Pauliblocking are ignored. It might be expected that the inclusion of the initial-state nucleon removal energy would affect the predictions. As statistical uncertainties are reduced with the next generation of long-baseline oscillation experiments, addressing these inconsistencies will become a focus of future development.

NEUT does not attempt to model a number of interaction channels that are irrelevant to SK and T2K analyses, these include neutrino-electron elastic scattering (used to provide complementary neutrino flux normalization constraints for higher energy beams [59]) and inverse beta decay (important for simulating reactor neutrino experiments).

\section{Additional tools}

So far we have focused on the implementation and physics models for simulation of neutrino-nucleus interactions. While this constitutes the core of the simulation, to be an effective tool for data analysis, NEUT provides a number of other tools and features.

neutgeom: The neutgeom tool is used to interface to a neutrino beamline simulation, perform neutrino raytracing, interface with a detector geometry description and correctly distribute interaction positions through the detector. Currently, neutgeom is only able to con- sume neutrino flux vectors from JNUBEAM, which simulates the J-PARC neutrino beam for the T2K experiment. There is some interest in distributing neutgeom as a standalone tool capable of interfacing with other neutrino beam simulations and interaction simulations.

NReWeight: Cross section reweighting is an important tool for systematic error estimation for neutrinoscattering analyses. For systematic parameters that can be effectively reweighted it enables interaction model variations to be applied at analysis time, rather than requiring re-simulation and re-analysis, and reduces the computing time taken to estimate systematic uncertainties by many many orders of magnitude. At its core, the reweighting technique calculates a weight for each already-simulated interaction,

$$
W=\frac{\sigma\left(p^{\prime}, \mathbf{x}\right)}{\sigma(p, \mathbf{x})}
$$

where $\mathbf{x}$ encapsulates the kinematics of the simulated interaction and $p$ and $p^{\prime}$ fully describe the model choices and any free parameters in the already-simulated and the varied model, respectively. This procedure is only exact when the varied model, $p^{\prime}$, predicts a range of $\mathbf{x}$ that is the same or a strict subset of the range predicted by $p$. NEUT provides the NReWeight package that implements exact cross section reweighting enabling the variation of nucleon form factors for CCQE and single meson production parameters after simulation. NReWeight also exposes reweighting for the meson and nucleon intranuclear cascade, which is exact for modest variations of the underlying meson-nucleon and nucleon-nucleon scattering probabilities. NReWeight is a critical tool for SK and T2K analyses.

Interface to GEANT3 and GEANT4: The simulation of an intranuclear cascade and of meson-nucleus and nucleon-nucleus scatterings are conceptually similar, the difference is whether the probe beings the simulation inside or outside of the simulated nucleus. As described in Sect. 3.3.1, the pion-nucleon interaction cross-sections were tuned to pion-nucleus scattering data. As a result, it is attractive for physics consistency to be able to simulate pion-nucleus scattering with the NEUT INC model. To achieve this, an interface between NEUT and GEANT was developed and integrated to the T2K near and SK detector simulation programs.

\section{Future direction}

Current and future neutrino experiments require precise neutrino interaction simulations to achieve their ambitious physics goals. To meet these requirements, NEUT will be continue to be developed and improved.

Current modelling improvements include the implementation of the state-of-the-art CCQE and multinucleon model by Amaro et al. [60], and single pion production models by Kabirnezhad [61] and by Sato et al. [62].The implementation of a rudimentary QE-only electron-scattering simulation in NEUT is underway. This 
will enable improved and extended validations of the implemented physics that is most critical to T2K analyses.

The dependence on CERNLIB is problematic, as the library is no longer maintained. Building and distributing the library for modern compilers and operating systems takes time away from more important development efforts. NEUT currently relies on CERNLIB for reading configuration files, random number generation and some common mathematical operations, and PYTHIA v5.72 for simulating SIS and DIS interactions. Resolving the dependence on PYTHIA v5 is not simple as changing to a newer version ( 66 or v8) would require the implementation of the particles' kinematics determination (vector generation) functions in NEUT, as the PYTHIA implementation that we rely on has been dropped or does not cover the entire kinematic region. Removing the dependency on CERNLIB is a high priority for near-future maintenance.

The current closed source nature of NEUT is undesirable. Exposure to more users and use cases will result in code, interface, and physics improvements. However, the lack of human resources render it difficult to support NEUT as a more general tool. Work has begun, in collaboration with other neutrino interaction simulation stake-holders, to define, test, and implement a new community-designed event format and event generation API [63]. These critical future developments will be implemented in NEUT as they become defined and mature.

\section{Summary}

NEUT is a general purpose neutrino interaction simulation used and improved by members of the SK and the T2K collaborations, with critical additional contributions from interaction theory groups. Future development will target the physics requirements of SuperKamiokande, T2K, and Hyper-Kamiokande, and software integration improvements to the NEUT API and data formats.

Acknowledgements The authors would like to thank the many contributors of NEUT, and the users in the SK, T2K, Ninja, and WAGASCI collaborations that push us to continue to develop it as a world-class analysis tool. The authors would also like to thank Kendall Mahn for help proofreading this document. This work is partially supported by MEXT/JSPS KAKENHI Grant Number 18H05536. This material is based upon work supported by the U.S. Department of Energy, Office of Science, Office under Award Number(s) DE-SC0015903.

Open Access This article is licensed under a Creative Commons Attribution 4.0 International License, which permits use, sharing, adaptation, distribution and reproduction in any medium or format, as long as you give appropriate credit to the original author(s) and the source, provide a link to the Creative Commons licence, and indicate if changes were made. The images or other third party material in this article are included in the article's Creative Commons licence, unless indicated otherwise in a credit line to the material. If material is not included in the article's Creative Commons licence and your intended use is not permitted by statutory regulation or exceeds the permitted use, you will need to obtain permission directly from the copyright holder. To view a copy of this licence, visit http://creativecomm ons.org/licenses/by/4.0/.

\section{References}

1. E.S. Pinzon Guerra et al., Phys. Rev. D 99, 052007 (2019)

2. M. Nakahata et al. [Kamiokande], J. Phys. Soc. Japan 55, $3786(1986)$

3. Y. Fukuda et al. [Super-Kamiokande], Phys. Rev. Lett. 81, 1562 (1998)

4. K. Abe et al. [Super-Kamiokande], Phys. Rev. D 97(7), 072001 (2018)

5. K. Abe et al. [T2K], Phys. Rev. D 91(7), 072010 (2015)

6. K. Abe et al. [T2K], Phys. Rev. D 87(1), 012001 (2013)

7. M. Honda, M. Sajjad Athar, T. Kajita, K. Kasahara, S. Midorikawa, Phys. Rev. D 92(2), 023004 (2015)

8. O. Benhar, N. Farina, H. Nakamura, M. Sakuda, R. Seki, Phys. Rev. D 72, 053005 (2005)

9. H. Ejiri, Phys. Rev. C 48, 1442 (1993)

10. S. Jadach, Z. Was, R. Decker, J.H. Kuhn, Comput. Phys. Commun. 76, 361-380 (1993)

11. C.H. Llewellyn Smith, Phys. Rep. 3C, 261 (1972)

12. R.A. Smith, E.J. Moniz, Nucl. Phys. B 43, 605 (1972)

13. R. Gran, J. Nieves, F. Sanchez, M.J. Vincente Vacas, Phys. Rev. D 88, 113007 (2013)

14. B. Bourguille, J. Nieves, F. Sanchez, JHEP 04, 004 (2021)

15. J. Nieves, J.E. Amaro, M. Valverde, Phys. Rev. C 70, 055503 (2004). [Erratum: Phys. Rev. C 72, 019902 (2005)]

16. O. Benhar, A. Fabrocini, S. Fantoin, I. Sick, Nucl. Phys. A 579, 493 (1994)

17. C. Juszczzak, J.A. Nowak, J.T. Sobczyk, Nucl. Phys. B Proc. Suppl. 159, 211 (2006)

18. A. Furmanski, University of Warwick, PhD Thesis No. $74036(2015)$

19. R. Bradford, A. Bodek, H.S. Budd, J. Arrington, Nucl. Phys. B Proc. Suppl. 159, 127 (2005)

20. A. Bodek, S. Avvakumov, R. Bradford, H.S. Budd, Eur. Phys. J. C 53, 349 (2008)

21. B. Bhattacharya, R.J. Hill, G. Paz, Phys. Rev. D 84, 073006 (2011)

22. A.S. Meyer, M. Betancourt, R. Gran, R.J. Hill, Phys. Rev. D 93, 113015 (2016)

23. C. Adamuscin, E. Tomasi-Gustafsson, E. Santopinto, R. Bijker, Phys. Rev. C 78, 035201 (2008)

24. J. Stowell, University of Sheffield, PhD Thesis No. 22623 (2019)

25. R. Gran et al. [K2K], Phys. Rev. D 74, 052002 (2006)

26. A.A. Aguilar-Arevalo et al. [MiniBooNE], Phys. Rev. D 88(3), 032001 (2013)

27. P. Adamson et al. [MINOS], Phys. Rev. D 91(1), 012005 (2015)

28. J. Marteau, Eur. Phys. J. A 5, 183 (1999) 
29. D. Rein, L.M. Sehgal, Ann. Phys. 133, 79 (1981)

30. C. Berger, L.M. Sehgal, Phys. Rev. D 76, 113004 (2007)

31. K.M. Graczyk, J.T. Sobczyk, Phys. Rev. D 77, 053003 (2008)

32. D. Rein, Z. C Phys. 35, 43 (1987)

33. T. Sjostrand, Comput. Phys. Commun. 82, 74 (1994)

34. M. Glück, E. Reya, A. Vogt, Eur. Phys. J. C 5, 461-470 (1998)

35. A. Bodek, U.K. Yang, AIP Conf. Proc. 792(1), 257 (2005)

36. S.J. Barish et al., Phys. Rev. D 17, 1 (1978)

37. B. JongeJans et al., Nuovo Cim. A 101, 435 (1989)

38. C. Bronner, M. Hartz, JPS Conf. Proc. 12, 010041 (2016)

39. H. Saarikko, Proceedings of 9th International Neutrino Conference, p. 507 (1979)

40. S. Barlag et al., Z. Phys. C 11, 283 (1982)

41. C. Berger, L.M. Sehgal, Phys. Rev. D 79, 053003 (2009)

42. D. Rein, L.M. Sehgal, Nucl. Phys. B 223, 29 (1983)

43. D. Rein, Nucl. Phys. B 278, 61 (1986)

44. D.S. Baranov et al., Yad. Fiz. 40, 1454 (1984)

45. V. Ammosov, Low multiplicity final states in neutrino interaction at low energy-SKAT results, NuINT01 workshop, KEK, Tsukuba, Japan (2001)

46. L.L. Salcedo, E. Oset, M.J. Vicente-Vacas, C. GarciaRecio, Nucl. Phys. A 484, 557-592 (1988)

47. E.S. Pinzon Guerra et al. (DUET Collaboration), Phys. Rev. C 95, 045203 (2017)

48. M.K. Jones et al., Phys. Rev. C 48, 2800 (1993)

49. D. Ashery et al., Phys. Rev. C 30, 946 (1984)

50. E. Bellotti, D. Cavalli, C. Matteuzzi, Nuovo Cim. A 18, $75(1975)$
51. E. Bellotti, S. Bonetti, D. Cavalli, C. Matteuzzi, Nuovo Cim. A 14, 567 (1973)

52. G. Rowe, M. Salomon, R.H. Landau, Phys. Rev. C 18, 584 (1978)

53. R. Seki, M. Masutani, Phys. Rev. C 27, 2799 (1983)

54. J.S. Hyslop, R.A. Arndt, L.D. Roper, R.L. Workman, Phys. Rev. D 46, 961 (1992)

55. B.R. Martin, M.K. Pidckco, Nucl. Phys. B 126, 285 (1977)

56. G.I. Lykasov, W. Cassing, A. Sibirtsev, M.V. Rzyanin, Eur. Phys. J. A 6, 71 (1999)

57. H.W. Bertini, Phys. Rev. C 6, 631 (1972)

58. S.J. Lindenbaum, R.M. Sternheimer, Phys. Rev. 105, 1874 (1957)

59. E. Valencia et al. [MINER $\nu$ A], Phys. Rev. D 100(9), 092001 (2019)

60. J.E. Amaro, M.B. Barbaro, J.A. Caballero, T.W. Donnelly, R. Gonzalez-Jimenez, G.D. Megias, I.R. Simo, arXiv:2106.02857 [hep-ph]

61. M. Kabirnezhad, Phys. Rev. D 102(5), 053009 (2020)

62. J.E. Sobczyk, E. Hernández, S.X. Nakamura, J. Nieves, T. Sato, Phys. Rev. D 98(7), 073001 (2018)

63. J. Barrow et al., FERMILAB-CONF-20-423-ND-SCD. arXiv:2008.06566 [hep-ex] 